\title{
MedienPädagogik
}

Zeitschrift für Theorie und Praxis der Medienbildung

Lernsituationen mit Metaphern und Wikibooks.

Fallstudien zu Entwicklungspotenzialen einer integrativen Medienbildung in der Lehrerinnen- und Lehrerbildung

\section{Fazit: Entwicklungspotenziale von Lernsituationen mit Metaphern und Wikibooks}

Franco Rau

\begin{abstract}
Zusammenfassung
In Form einer entwicklungsorientierten Bildungsforschung erfolgte in den vorangegangen Kapiteln die Problematisierung der aktuellen Praxis zur integrativen Medienbildung in den erziehungswissenschaftlichen Studienanteilen des Lehramtsstudiums, der Entwurf einer neuen Praxis, die empirische Erprobung und wissenschaftliche Analyse des Entwurfs sowie eine zusammenfassende Interpretation der gewonnenen Erkenntnisse. In zwei Iterationen erfolgte eine systematische Untersuchung der Entwicklungspotenziale von Lernsituationen mit Metaphern und Wikibooks. Das abschließende Kapitel bündelt die Vielzahl der gewonnen Erkenntnisse der gesamten Arbeit. Zur Beantwortung der Forschungsfrage wird gezeigt, wie durch die Mitgestaltung eines öffentlichen Wikibooks in erziehungswissenschaftlichen Seminaren Situationen geschaffen werden können, um eine pädagogische Artikulations- und Reflexionsfähigkeit mit Metaphern (weiter) zu entwickeln und ein Lernen mit und über soziale Medien erfahrungsorientiert anzuregen und zu unterstützen.
\end{abstract}

12. Fazit

«Eine als entwicklungsorientiert konzipierte Bildungsforschung würde den Versuch machen, Theorie, Modellierung von Praxis, hermeneutische Verständigung, empirische Erprobung, wissenschaftliche Evaluation und theoriegeleitete Reflexion aufeinander zu beziehen und voneinander abhängig zu machen. Ziel solcher rückgekoppelter Prozesse wären Erkenntnisse sowohl über die bestehende Realität als auch über Formen, Elemente und Bedingungen prozessualer Erschließung von Veränderungspotenzialen - und damit auch Veränderung der Bildungspraxis. Bildungsinnovation wäre ein genuiner Bestandteil einer so verstandenen Entwicklungsforschung (versus nachträgliche Transferförderung wissenschaftlicher Erkenntnisse)» (Sesink und Reinmann 2015, 70).

Der methodologische Ausgangspunkt des vorliegenden Projektes war ein gestaltungs- und entwicklungsorientiertes Verständnis von Bildungsforschung. Es wurde davon ausgegangen, dass erziehungs- und bildungswissenschaftliche Fragestellungen nicht im Stil naturwissenschaftlicher Forschung beantwortet werden können, da der spezifische Charakter des Gegenstandsbereiches dieser Disziplinen auch 
spezifische Herausforderungen beinhaltet (Kap. 2.1). Der jeweilige Kontext von Vermittlungssituationen sowie die Komplexität pädagogischer Interaktionszusammenhänge limitieren einerseits die Möglichkeit, verallgemeinernde Schlüsse ziehen zu können und benötigen andererseits eine spezifische Forschungsmethodik, um gegenstandsangemessene Erkenntnisse zu gewinnen (Berliner 2002). Zum Umgang mit diesen ausgewählten Herausforderungen wird in gestaltungs- und entwicklungsorientierten Forschungsansätzen der Versuch unternommen, wie es im Zitat von Sesink und Reinmann $(2015,70)$ für das Konzept der entwicklungsorientierten Bildungsforschung zum Ausdruck gebracht wird, Wissenschaft und Praxis wechselseitig aufeinander zu beziehen (Kap. 2.2). An dieses Verständnis anknüpfend, erfolgte die Untersuchung von Veränderungs- und Entwicklungspotenzialen pädagogischer Praxis zur integrativen Medienbildung für das Handlungsfeld erziehungswissenschaftlicher Seminare im Lehramtsstudium in Form einer entwicklungsorientierten Bildungsforschung (Kap. 3).

Mit der Formulierung einer integrativen Medienbildung wird in Anlehnung an den bildungspolitischen Diskurs zum Ausdruck gebracht, dass die medienpädagogische Professionalisierung im Lehramtsstudium potenziell als integraler Bestandteil aller Studienfächer betrachtet wird (Kap. 1.1, 4.2.1.3). Die Vermittlung fachspezifischer Fähigkeiten soll - der Logik bildungspolitischer Leitbilder folgend - durch ein Lernen mit innovativen digitalen Medien erfolgen und zugleich ein Lernen über digitale Medien ermöglichen (Kap. 4.2.1.2). Das Ziel des vorliegenden Projektes war in diesem Zusammenhang die Untersuchung der Fragestellung, inwiefern die Mitgestaltung an der öffentlichen Sharing-Community Wikibooks im Kontext erziehungswissenschaftlicher Seminare Entwicklungspotenziale für eine integrative Medienbildung im Lehramtsstudium eröffnen kann. Der Begriff Entwicklungspotenziale umfasste in Anlehnung an den Entwicklungsbegriff von Sesink und Reinmann (2015) drei Bedeutungsdimensionen. In der ersten Dimension wurde Entwicklung als didaktische Gestaltung von Lehr- und Lernsituationen mit sozialen Medien in Seminaren im Lehramtsstudium betrachtet. Die zweite Dimension umfasste das Verständnis von Entwicklung als Bildungsgeschehen bzw. Lernhandlungen der Studierenden zur Aneignung einer pädagogischen Artikulations- und Reflexionsfähigkeit sowie der Fähigkeit zur Teilhabe an einer partizipativen Medienkultur. In der dritten Bedeutungsdimension wurde Entwicklung als reflexiver und partizipativer Prozess aller Beteiligten am Forschungs- und Gestaltungsprozess der entworfenen Lehr- und Lernsituationen zur Ermöglichung von Lernhandlungen gefasst.

Die Bearbeitung der Fragestellung in Form eines entwicklungsorientierten Forschungs- und Praxisprojektes erfolgte anknüpfend an das von mehreren Autorinnen und Autoren formulierte Plädoyer für mehr praxisorientierte Forschung in der deutschsprachigen Medienpädagogik (z. B. Spanhel 2007; Petko 2011; Niesyto 2014). Die Fragestellung beinhaltete neben dem praxisbezogenen Bezug zum aktuellen 
bildungspolitischen Diskurs zur Medienbildung als institutionellem Bildungsauftrag (Kap. 4.2.1) vielfältige Bezüge zu verschiedenen wissenschaftlichen Diskussions- und Themenfeldern. In Anlehnung an Petko (2011) erfolgte die Verknüpfung einer medienpädagogischen und einer mediendidaktischen Perspektive, indem über die innovative Gestaltung von Lehr- und Lernsituationen mit sozialen Medien untersucht wurde, inwiefern Studierende für eine Teilhabe an öffentlicher Wissensproduktion mit Wikis - im Sinne von partizipativen Medienkulturen (Biermann et al. 2014) - sensibilisiert werden können. Zur Verortung in den jeweiligen Diskursen wurde u. a. eine begrifflich-theoretische Positionierung vorgenommen (Kap. 4.3), mediendidaktische Forschungsergebnisse über die institutionelle Verwendung sozialer Medien in der Hochschullehre aufgearbeitet (Kap. 4.4) und es erfolgte eine Darstellung medienpädagogischer Prinzipien zur handlungsorientierten Medienerziehung (Kap. 4.5.1). Ferner wurde argumentiert, inwiefern die Fähigkeit zur Teilnahme und Teilhabe an einer partizipativen Kultur als Aspekt einer medienpädagogischen Kompetenz verstanden werden kann (Kap. 4.5.2.1).

Zur Analyse des fachlichen Lernens in erziehungswissenschaftlichen Seminaren wurde die Frage untersucht, inwiefern die Auseinandersetzung mit Metaphern Entwicklungspotenziale für eine pädagogische Artikulations- und Reflexionsfähigkeit eröffnen kann. Diese Frage basiert auf der Auseinandersetzung mit Forschungsergebnissen zu erfahrungsfundierten Vorstellungen von Studierenden hinsichtlich erziehungswissenschaftlicher Begriffe und Phänomene, welche in Anlehnung an Blömeke (2004) als Lernvoraussetzungen betrachtet wurden (Kap. 5). Im Zusammenhang mit dem Leitbild und Diskussionsthema einer reflexiven Lehrerinnen- und Lehrerbildung wurde auf Basis einer begrifflich-theoretischen Diskussion unterschiedlicher Konstrukte die Entscheidung getroffen (Kap. 5.3), Metaphern bzw. metaphorische Konzepte als potenzielles Reflexionsinstrument und als Forschungsgegenstand zu berücksichtigen. Dieses Vorgehen umfasste zum einen die Aufarbeitung theoretischer und empirischer Forschungsergebnisse sowie aktueller Diskussionen über Metaphern in der Lehrerinnen- und Lehrerbildung (Kap. 5.4). Zum anderen wurden Metaphern als didaktisches Vermittlungsinstrument und als Forschungsgegenstand in der Pädagogik diskutiert (Kap. 5.5).

Auf Basis der praxisbezogenen Problematisierung sowie unter Berücksichtigung des skizzierten Forschungsstandes wurde zur Untersuchung der formulierten Fragestellung ein theoretisch begründeter Seminarentwurf entwickelt (Kap. 6). Mit dem Entwurf sollte Studierenden die Möglichkeit eröffnet werden, eine pädagogische Artikulations- und Reflexionsfähigkeit zu entwickeln und sich die Fähigkeit zur Teilhabe an einer partizipativen Medienkultur anzueignen (Kap. 6.2). Darüber hinaus wurde mit dem Entwurf das Ziel verfolgt, dass Studierenden neue Möglichkeiten zur Verwendung und Implementierung digitaler (sozialer) Medien in institutionellen Vermittlungssituationen erfahren. Der Entwurf orientierte sich u. a. an 
den medienpädagogischen und mediendidaktischen Prinzipien der «Produkt- und Projektorientierung» (Kap. 6.5.1), der «Kooperation, Kollaboration und Kommunikationsorientierung» (Kap. 6.5.2) und zielt auf die «Eröffnung von Partizipationsmöglichkeiten und Erfahrungsorientierung» (Kap. 6.5.3) ab. So wurde ein dreiphasiges Blended-Learning-Konzept zur kollaborativen Erstellung eines Wikibooks mit Studierenden entworfen (Kap. 6.6).

Dieses Konzept wurde als universitäre Lehrveranstaltung in den Modulen «Didaktik, Methodik und Medien» und «Grundlagen pädagogischen Denkens und Handelns» in einem zirkulären Vorgehen über zwei Semester an der TU Darmstadt erprobt, analysiert, modifiziert und hinsichtlich der formulierten Fragestellung ausgewertet. Die Erprobung, Analyse und Auswertung umfasste vier Seminare mit insgesamt $111 \mathrm{Stu}$ dierenden. Die Ergebnisse dieses zirkulären Vorgehens sind vielschichtig und können im Folgenden nur akzentuierend zusammengefasst werden. Zur Beantwortung der Forschungsfrage werden fünf Aspekte hervorgehoben:

- Medienbezogene Lern- und Reflexionsanlässe durch die Mitgestaltung eines Wikibooks: Die gemeinsame Erstellung eines öffentlichen Wikibooks mit Studierenden kann im Rahmen institutioneller Lehrveranstaltungen verschiedene Anlässe zur Entwicklung der Fähigkeit zur Teilhabe an einer partizipativen Medienkultur eröffnen und damit zur medienpädagogischen Professionalisierung im Lehramtsstudium beitragen (Kap. 11.3, 11.4). Diese Anlässe umfassen u. a. (a) das Gestalten und Veröffentlichen eigener Wikibeiträge, (b) das Diskutieren und Beurteilen von Bedingungen der öffentlichen Wissensproduktion im Kontext der Wikibookgemeinschaft sowie (c) die Auswahl und Nutzung von sozialen Medien zur Kooperation und zum Lernen. Die Ermöglichung sowie die Wahrnehmung dieser Anlässe wurden im Rahmen des Projektes durch verschiedene Faktoren und didaktische Entscheidungen beeinflusst: (1.) dem Grad der didaktischen Vorstrukturierung, (2.) der Vorstellung eines Wikibooks von der Lehrperson sowie (3.) von Gruppenprozessen und der Gruppenzusammenstellung der Seminarteilnehmerinnen und -teilnehmer (Kap. 11.3.2).

- "Kein 0815-Seminar», Projektseminare mit Wikibooks als neuer Erfahrungsraum zum Lernen mit sozialen Medien: Die Durchführung eines Seminars mit Projektcharakter zur kollaborativen (Mit-)Gestaltung eines Wikibooks kann Studierenden neue Erfahrungen zur Verwendung digitaler Medien in institutionellen Lehrveranstaltungen sowie im Rahmen ihres Studiums ermöglichen. Nahezu alle befragten Studierenden brachten entsprechende Erfahrungen und Eindrücke über die verschiedenen Erhebungsmethoden zum Ausdruck. Diese Erfahrungen können zum Ausgangspunkt werden, um die Selbstverständlichkeiten zur Gestaltung institutionalisierter Lehre sowie zur Verwendung von Medien im Unterricht zu reflektieren (Kap. 8.3.1, 11.4). Interessant ist zum einen, dass eine solche Reflexion auch von Studierenden zum Ausdruck gebracht wurde, die nur peripher (bzw. nicht aktiv 
den Wiki-Editor nutzend) an der Gestaltung des Wikibooks mitwirkten. Zum anderen wurde von Studierenden auch in Veranstaltungen, in denen gemäss Modulbeschreibungen die Auseinandersetzung mit Medien kein vorgegebener Lehr- bzw. Lerninhalt war, der Wunsch formuliert, die Einsatzmöglichkeiten von Wikis im Unterricht zu thematisieren (Kap. 8.3.1).

- Metaphern als herausfordernder Anlass zur Artikulation und Reflexion eigener Vorstellungen zum Lehren und Lernen: Die Formulierung von Metaphern im Rahmen der Seminare stellte für Studierende einen herausfordernden Anlass zur Artikulation eigener und gruppenspezifischer Vorstellungen dar (Kap. 8.2, 11.2). Die entstandenen Metaphern offenbaren eine Vielfalt an originellen Ausdrucksweisen über Lehren und Lernen. Die individuellen Ad-hoc-Metaphern der Studierenden konnten zu acht verschiedenen metaphorischen Mustern verdichtet werden (Kap. 11.2.1). Die rekonstruierten Muster bringen jeweils unterschiedliche Vorstellungen vom Lehren und Lernen zum Ausdruck. Das metaphorische Muster «Lehren und Lernen ist GARTENARBEIT UND WACHSTUM» umfasst beispielsweise Formulierungen von Studierenden, die Lernen als einen individuellen (Wachstums-)Prozess beschreiben, der durch die Gestaltung relevanter Bedingungen (in Form von Gartenarbeit) unterstützt werden kann (Kap. 7.4.3.2, 10.4.3.2). Im Rahmen des metaphorischen Musters "Lehren ist MATERIALBEARBEITUNG (UND OBJEKTBEWEGUNG)» werden Lernende hingegen als bearbeitbare bzw. bewegbare Objekte konzeptualisiert, welche nach den Vorstellungen von Lehrenden gestaltet bzw. verändert werden können (Kap. 7.4.3.2, 10.4.3.2). Die vergleichende Betrachtung dieser unterschiedlichen Ad-hoc-Metaphern und Gruppenmetaphern ermöglicht vielfältige Diskussions- und Reflexionsanlässe. Diese Anlässe eröffnen sich u. a. hinsichtlich der metaphorisierten Rollenbilder von Lehrenden und Lernenden, der Verwendung einer geschlechtergerechten Sprache sowie hinsichtlich der Bedeutung von Medien in Vermittlungssituationen (Kap. 8.2, 11.2). In der Annahme, dass die Studierenden mit den formulierten Metaphern ihre - sprachlich artikulierbaren - Vorstellungen zum Ausdruck bringen (Kap. 5.3.3), eröffnen sich zudem Relationierungsmöglichkeiten der eigenen Konzepte zu pädagogischen und didaktischen Modellen. Auf Basis der vorgestellten empirischen Ergebnisse kann davon ausgegangen werden, dass die Formulierung und Diskussion von Metaphern im Rahmen der entworfenen Seminarkonzepte zur Entwicklung einer pädagogischen Artikulations- und Reflexionsfähigkeit beitragen kann.

- Zwischen Entwicklungspotenzialen und Bewältigungsstrategien: Die zuvor skizzierten Ergebnisse hinsichtlich der medienpädagogischen Professionalisierungsmöglichkeiten, der potenziellen Erfahrungen zur Gestaltung institutioneller Lehre sowie der Artikulations- und Reflexionsanlässe durch Metaphern markieren empirisch begründete Entwicklungspotenziale. Diese Ergebnisse eröffnen potenzielle Handlungs- und Gestaltungsspielräume auf einer «mikrosozialen Ebene» 
(Kron et al. 2014, 45) bzw. auf den hochschuldidaktischen Handlungsebenen der «(Lern)Situationen» und «(Lehr)Veranstaltungen» (Wildt 2002, 8). Die empirische Erprobung zeigte jedoch, dass diese Potenziale nicht von allen Studierenden als Möglichkeit bzw. Impuls für individuelle Entwicklungen und Lernhandlungen wahrgenommen wurden. Für die entwickelten Lernsituationen wurden im Rahmen der Analysen und Auswertungen verschiedene - aus subjektiver Perspektive durchaus nachvollziehbare - Bewältigungsstrategien von Studierenden sichtbar (Kap. 11.5).

- Entgrenzungsphänomene beim Lernen mit sozialen Medien als Forschungslücke der Didaktik: Im Rahmen des Forschungsstandes wurde skizziert, dass sozialen Medien u. a. das Potenzial zugeschrieben wird, die Grenzen institutionalisierter Lehre zu verschieben bzw. öffnen zu können (Kap. 4.4.2). Diese Potenziale konnten über die (Mit-)Gestaltung eines öffentlichen Wikibooks im Rahmen eines Seminars für Studierende auf unterschiedlichen Ebenen erfahrbar werden, z. B. durch die Bearbeitung der erstellten Beiträge von Wikibookianerinnen und Wikibookianer sowie in Form expliziter Diskussionskommentare (Kap. 7.2.2.3, 8.3.4, 10.2.3.3, 11.3.1.2). Die Einbindung von sozialen Medien im Sinne einer offenen Sharing-Community (Kap. 4.3.3.3) in institutionelle Lehrveranstaltungen ermöglichte Interaktionen mit Menschen ausserhalb der Institution und wurde zum Ausgangspunkt verschiedener Diskussionen und Reflexionen über die Teilhabe an einer partizipativen Medienkultur (Kap. 8.3.4, 11.3). Zugleich zeigte sich, dass die Interaktionen über die gewählte Plattform Wikibooks nur mit wenigen Mitgliedern der Wikicommunity erfolgten und in den zwei Iterationen zu unterschiedlichen Erfahrungen der Teilnehmerinnen und Teilnehmern führten. Diese Entgrenzungsphänomene werden in didaktischen Planungsmodellen, welche (digitale) Medien als Mittel und Werkzeuge zur Erreichung spezifischer Ziele konzeptualisieren bzw. metaphorisieren ${ }^{322}$, kaum adäquat modelliert. Darüber hinaus konnten für das Modell der partizipativen Mediendidaktik von Mayrberger (2013a, 2014) mögliche Anschlussstellen und neue Perspektiven diskutiert werden (Kap. 11.4.3).

Die hervorgehobenen Erkenntnisse sind das Ergebnis eines entwicklungsorientierten Bildungsforschungsprojektes mit einem spezifischen Erkenntnisinteresse und entsprechenden Geltungsgrenzen. Statt «feststellende Aussagen» (Sesink und Reinmann 2015, 82) mit einem Verallgemeinerungsanspruch zu generieren, dient der gewählte Forschungsansatz insbesondere dem Ziel, Erkenntnisse über bestehende und neue Entwicklungspotenziale einer innovativen Bildungspraxis zu gewinnen. Das vorgestellte Projekt zur Bestimmung der Entwicklungspotenziale einer integrativen Medienbildung für das Handlungsfeld erziehungswissenschaftlicher Seminare

322 Dies gilt u. a. für Modelle der Allgemeinen Didaktik, der Hochschuldidaktik, der Mediendidaktik sowie für eine Vielzahl an Fachdidaktik. 
im Lehramtsstudium kann methodologisch als «Fallstudie» bezeichnet werden. Zur Sicherung der methodologischen Angemessenheit und forschungsmethodischen Qualität der durchgeführten Fallstudie erfolgte eine Berücksichtigung der von Tulodziecki et al. $(2013,230)$ vorgeschlagenen Qualitäts- und Prozessstandards zur Durchführung gestaltungsorientierter Bildungsforschung (Kap. 2.2) sowie die Beachtung der methodischen Gütekriterien für Sozialforschung von Steinke (2012) (Kap. 8.4, 11.6). Argumente zur Begründung sowie zur Relevanz gestaltungs- und entwicklungsorientierter Forschung wurden in der vorliegenden Arbeit ausführlich präsentiert und diskutiert (Kap. 2). Exemplarisch lassen sich an dieser Stelle zwei Argumente hervorheben. Zum einen ist es die Praxisrelevanz der gewonnenen Erkenntnisse für die integrative Medienbildung in der Lehrerinnen- und Lehrerbildung (Kap. 4.2.1.4). Die Entwicklung von innovativer Hochschullehre mit digitalen sozialen Medien wurde im Rahmen des gewählten Forschungsansatzes zu einem zentralen Gegenstand der wissenschaftlichen Analyse. Die Erkenntnisse über theoretisch und empirisch begründeten Entwicklungspotenziale können Hochschullehrenden neue Handlungsspielräume eröffnen und präsentieren potenzielle Wirkungen medienpädagogischen Handelns. Über den wechselseitigen Bezug von Theorie, Praxis und empirischer Forschung, wie im Zitat von Sesink und Reinmann (2015) am Anfang des Kapitels dargestellt, eröffnen sich zum anderen neue Perspektiven für bildungswissenschaftliche Forschung und Theorieentwicklung. Dies wurde akzentuierend für die Berücksichtigung von Entgrenzungsphänomenen institutioneller Lehre durch soziale Medien für didaktische Planungs- und Handlungsmodelle markiert (Kap. 11.4.3).

Das Ziel dieser Arbeit war es, einen Beitrag zur Gestaltung und Analyse von Veränderungs- und Entwicklungspotenzialen einer integrativen Medienbildung im Lehramtsstudium zu leisten. In einem weiten Verständnis von Integration wurde davon ausgegangen, dass die erziehungs- und bildungswissenschaftlichen Studienanteile in der ersten Phase der Lehrerinnen- und Lehrerbildung Erfahrungsräume, in denen das Lernen mit und über Medien einen integrativen Bestandteil darstellt, schaffen müssen. In Form eines entwicklungs- und gestaltungsorientierten Bildungsforschungsprojektes konnten auf den hochschuldidaktischen Handlungsebenen der Lehrveranstaltungen und Lernsituationen neue Handlungsspielräume entwickelt und deren Entwicklungspotenziale empirisch begründet werden. In Anlehnung an die am Anfang der vorlegten Arbeit zitierte Aufgabenstellung der Arbeitsgruppe Erziehungswissenschaft (1997) erfolgte dabei eine Berücksichtigung unterschiedlicher Perspektiven: die Nutzung von Metaphern als Reflexions- und Forschungsinstrument in Seminaren der erziehungswissenschaftlichen Studien im Lehramt aus erziehungswissenschaftlicher Perspektive, die medien- und hochschuldidaktische Entwicklung und Analyse von Lehr- und Lernarrangements zur Unterstützung kollaborativer und reflexiver Lernprozesse mit sozialen Medien sowie die aus medienerzieherischer Perspektive begründete Ermöglichung neuer medialer Erfahrungsräume zur 
handlungsorientierten Teilhabe an Wikibooks als Beispiel für partizipative Medienkulturen. So bietet die als Fallstudie zu bewertende Arbeit einen umfassenden Beitrag für das komplexe Handlungs- und Forschungsfeld der integrativen Medienbildung.

\section{Literatur}

Arbeitsgruppe Erziehungswissenschaft, Bardo Herzig. 1997. «Arbeitsgruppe «Erziehungswissenschaft»». In Neue Medien - neue Aufgaben für die Lehrerausbildung: Tagungsdokumentation, herausgegeben von Gerhard Tulodziecki und Sigirid Blömeke, 39-53. Gütersloh: Verlag Bertelsmann Stiftung.

Berliner, David C. 2002. "Comment: Educational Research:The Hardest Science of All». Educational Researcher 31 (8): 18-20. https://doi.org/10.3102/0013189X031008018.

Biermann, Ralf, Johannes Fromme, und Dan Verständig. 2014. «Partizipative Medienkulturen als Transformation von Beteiligungsmöglichkeiten - Einleitung». In Partizipative Medienkulturen: Positionen und Untersuchungen zu veränderten Formen öffentlicher Teilhabe, herausgegeben von Ralf Biermann, Johannes Fromme, und Dan Verständig, 7-17. Wiesbaden: Springer Fachmedien Wiesbaden. https://doi.org/10.1007/978-3-658-01793-4_1.

Blömeke, Sigrid. 2004. «Empirische Befunde zur Wirksamkeit der Lehrerbildung». In Handbuch Lehrerbildung, herausgegeben von Sigrid Blömeke, Peter Reinhold, Gerhard Tulodziecki, und Johannes Wildt, 59-91. Bad Heilbrunn: Julius Klinkhard Verlag.

Kron, Friedrich W., Jutta Strandop, und Jürgens Eiko. 2014. Grundwissen Didaktik. 6. Auflage. München, Basel: Reinhardt.

Mayrberger, Kerstin. 2013. «Eine partizipative Mediendidaktik (nicht nur) für den Hochschulkontext?» In E-Learning zwischen Vision und Alltag. Zum Stand der Dinge, herausgegeben von C. Bremer und D. Krömker, 96-106. Münster [u. a.]: Waxmann. https://www.waxmann. com/?elD=texte\&pdf=2953Volltext.pdf\&typ=zusatztext.

Mayrberger, Kerstin. 2014. «Partizipative Mediendidaktik. Inwiefern bedarf es im Kontext einer partizipativen Medienkultur einer spezifischen Mediendidaktik?» In Partizipative Medienkulturen: Positionen und Untersuchungen zu veränderten Formen öffentlicher Teilhabe, herausgegeben von Ralf Biermann, Johannes Fromme, und Dan Verständig, 261-282. Wiesbaden: Springer Fachmedien Wiesbaden. https://doi.org/10.1007/978-3-658-01793-4_12.

Niesyto, Horst. 2014. «Medienpädagogische Praxisforschung». In Jahrbuch Medienpädagogik 10: Methodologie und Methoden medienpädagogischer Forschung, herausgegeben von Anja Hartung, Bernd Schorb, Horst Niesyto, Heinz Moser, und Petra Grell, 173-191. Wiesbaden: Springer Fachmedien Wiesbaden. https://doi.org/10.1007/978-3-658-04718-4_9.

Petko, Dominik. 2011. «Praxisorientierte medienpädagogische Forschung: Ansätze für einen empirischen Perspektivenwechsel und eine stärkere Konvergenz von Medienpädagogik und Mediendidaktik». MedienPädagogik: Zeitschrift für Theorie und Praxis der Medienbildung 20 (0): 245-258. https://doi.org/10.21240/mpaed/20/2011.09.22.X. 
Sesink, Werner, und Gabi Reinmann. 2015. «Umrisse eines Strukturmodells für entwicklungsorientierte bildungswissenschaftliche Forschung». In Entwicklungsorientierte Bildungsforschung. Plädoyer für einen ‘dritten Weg) in pädagogischer Forschung. Eine Textsammlung, herausgegeben von Werner Sesink, 69-83. http://www.sesink.de/wordpress/wp-content/ uploads/2015/11/Entwicklungsorientierte-Bildungsforschung_Sesink_2015.pdf.

Spanhel, Dieter. 2007. «Zur Standortbestimmung der Medienpädagogik aus anthropologischer und bildungswissenschaftlicher Sicht». In Jahrbuch Medien-Pädagogik 6: Medienpädagogik- Standortbestimmung einer erziehungswissenschaftlichen Disziplin, herausgegeben von Werner Sesink, Michael Kerres, und Heinz Moser, 33-54. Wiesbaden: VS Verlag für Sozialwissenschaften. https://doi.org/10.1007/978-3-531-90544-0_2.

Steinke, Ines. 2012. «Gütekriterien qualitativer Forschung». In Qualitative Forschung: ein Handbuch, herausgegeben von Uwe Flick, Ernst von Kardorff, und Ines Steinke, 319-331. Reinbek bei Hamburg: Rowohlt-Taschenbuch-Verl.

Tulodziecki, Gerhard, Silke Grafe, und Bardo Herzig. 2013. Gestaltungsorientierte Bildungsforschung und Didaktik: Theorie - Empirie - Praxis. Bad Heilbrunn: Klinkhardt.

Wildt, Johannes. 2002. «Ein hochschuldidaktischer Blick auf Lehren und Lernen». In Neues Handbuch Hochschullehre, herausgegeben von Brigitte Berendt, Hans-Peter Voss, und Johannes Wildt, 1-10. Bonn: Raabe-Verlag. 